> Véritables agents de surveillance et d'intervention lors des réponses anti-infectieuses et antitumorales, les lymphocytes $T$ obéissent à des règles strictes de conduite via la stimulation de leurs récepteurs membranaires. En effet, la reconnaissance antigénique est assurée par le récepteur à l'antigène $(T c R)$, appelé signal 1 , et les molécules de costimulation optimisent les signaux transmis (signal 2), permettant l'activation optimale des lymphocytes T. Parmi ces molécules de costimulation, certaines vont induire des signaux inhibiteurs. La plus emblématique est la molécule d'adhérence CTLA-4 (cytotoxic T-lymphocyte antigen-4). Entre découverte historique et immunothérapie prometteuse, cette revue décrit les mécanismes moléculaires de freinage mis en œuvre par cette molécule qui justifient son intérêt en termes de cible thérapeutique. <

La régulation des réponses immunes dépend de deux familles de gènes : la superfamille du TNF (tumor necrosis factor)/TNF- $R$ ( $R$ pour récepteur) et celle des immunoglobulines (IgSF). Ces molécules interagissent avec des ligands présents à la surface cellulaire qui régulent les fonctions immunes de façon positive (costimulation) ou négative (co-inhibition). Elles contrôlent donc les réponses contre les agents pathogènes, les tumeurs ainsi que les réponses aux antigènes du soi (auto-immunité).

Dans le cas de la stimulation des lymphocytes $T$, la reconnaissance antigénique est assurée par le récepteur pour l'antigène (TcR) - appelé signal 1 - et les molécules de costimulation optimisent les signaux transmis (signal 2). Ces notions ont été modélisées par le groupe de R. Schwartz dans des clones T murins [1]. Parmi ces molécules de costimulation de la famille des Ig (IgSF), CD28 reste la protéine emblématique qui permet en particulier la costimulation de cellules $\mathrm{CD}^{+}$naïves. Les ligands de CD28, CD80 et CD86, appartiennent à la famille B7 et sont présents sur les cellules présentatrices d'antigène. Cytotoxic T-lymphocyte antigen-4 (CTLA-4, CD152) a été identifiée comme étant

\title{
Rôle de CTLA-4 dans la cosignalisation négative du système immunitaire
}

\author{
Daniel Olive, Suong le Thi, Luc Xerri, \\ Ivan Hirsch, Jacques A. Nunès
}

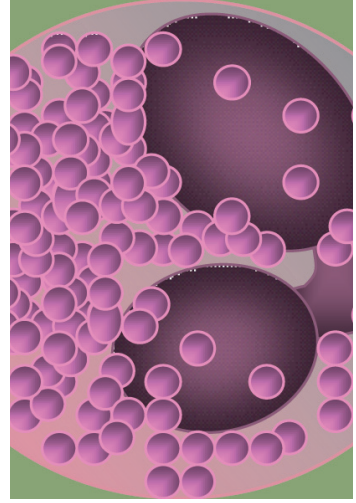

une molécule qui partage les mêmes ligands que CD28 mais exerce des fonctions inhibitrices. Les données actuelles convergent pour indiquer que son rôle essentiel est d'inhiber les fonctions de costimulation relayées par CD28 bien que des effets directs de CTLA-4 soient démontrés.

CTLA-4 est la première cible appartenant à la famille des molécules de cosignalisation qui soit entrée dans l'ère thérapeutique et dont les succès récents sont décrits par $C$. Robert et $C$. Matteus dans ce numéro de médecine/sciences [37]. Cette stratégie d'« inhibition des inhibiteurs » inaugure un nouveau concept thérapeutique et, de ce fait, une nouvelle classe de molécules thérapeutiques est née, utilisée en particulier en cancérologie (Figure 1).

\section{CD28 : un accélérateur (costimulateur)}

En l'absence de mécanisme de costimulation (par exemple via CD28), la stimulation par le TcR se traduit souvent par une non-réponse, un état d'anergie ${ }^{1}$ ou la mort cellulaire [2]. Le signal de costimulation a pour caractéristique essentielle de permettre une réponse immune même lorsque la stimulation antigénique est suboptimale. La costimulation par CD28 augmente la production de cytokines, en particulier

${ }^{1}$ L'anergie est caractérisée par l'incapacité de répondre normalement à des signaux d'activation. 


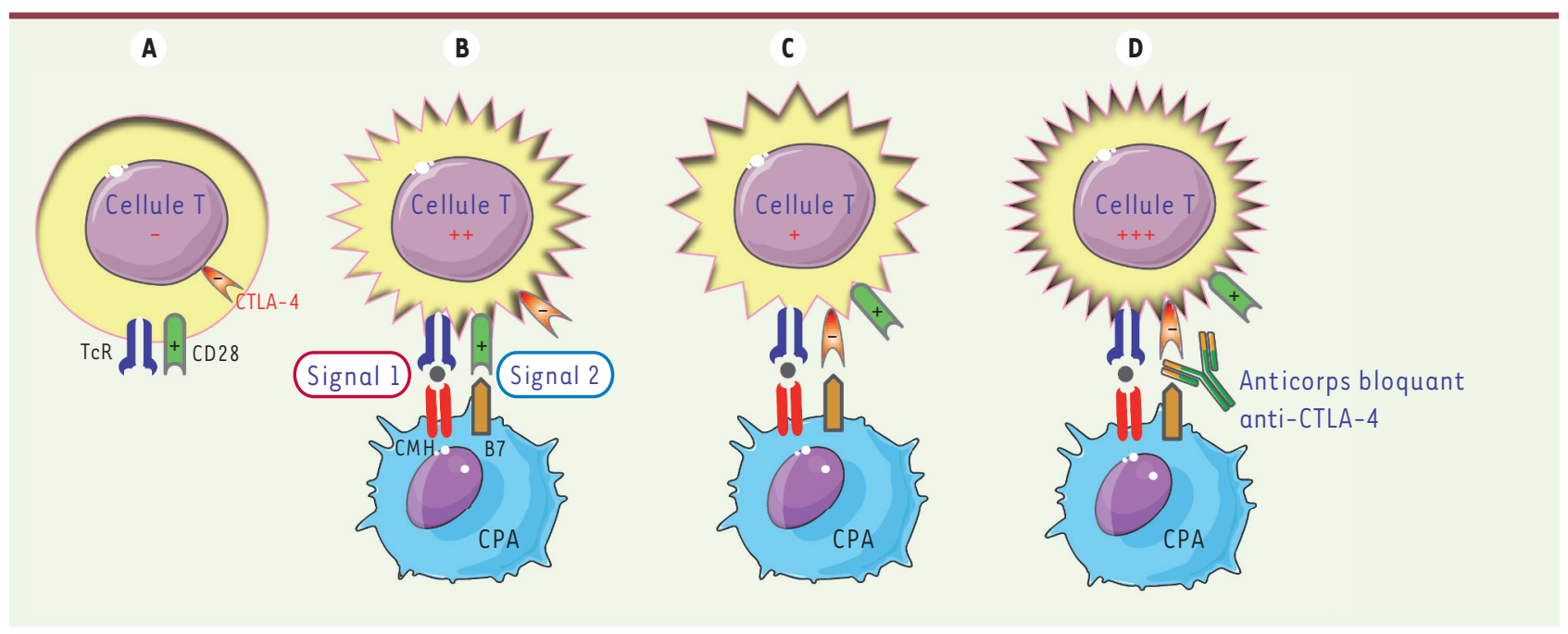

Figure 1. Fonctions immunorégulatrices de CTLA-4. En A, dans le lymphocytes au repos, CTLA-4 est intracytoplasmique. Elle apparaît à la surface cellulaire des lymphocytes T conventionnels après leur activation (B-D). L'activation T résulte de la fixation de l'antigène (présenté par le $C M H$ ) au TcR (signal 1) et de l'intervention de molécules de costimulation (CD28 liée aux ligands B7). CTLA-4 partage les mêmes ligands que CD28, et lorsqu'il s'y fixe, entraîne une inhibition de l'état d'activation T (C). La neutralisation de CTLA-4 par un anticorps (D) accroît l'activation T en privilégiant la survenue du signal 2 de costimulation via CD28. CMH : complexe majeur d'histocompatibilité ; TcR : T-cell receptor; CPA : cellule présentatrice d'antigène; CTLA-4 : cytotoxic T-lymphocyte antigen-4.

celle d'interleukine (IL)-2, l'expression du récepteur de l'IL-2 [3-5], la survie cellulaire via l'expression accrue des molécules anti-apoptotiques dont $\mathrm{BCl}-\mathrm{X}_{\mathrm{L}}[6]$. II existe des signaux activés par CD28 indépendamment de l'activation antigénique comme cela a été démontré par l'utilisation d'anticorps monoclonaux (Acm) anti-CD28 nommés superagonistes [7].

L'analyse des mécanismes transcriptionnels et post-transcriptionnels régulés par CD28 a permis de démontrer que cette molécule contrôle l'expression et l'activité des facteurs de transcription NFAT (nuclear factor of activated T-cells), NF-kB et AP-1 sur le promoteur du gène de I'IL-2 [8, 9].

La partie intracytoplasmique (IC) de CD28 (41 acides aminés chez l'homme) ne comporte aucune activité enzymatique démontrée, mais l'analyse de la séquence peptidique a permis d'y identifier des motifs qui lui permettent de s'associer à des enzymes et des protéines adaptatrices impliquées dans la transmission du signal intracellulaire. Parmi les motifs les plus importants, on peut citer YMNM qui rend compte, après la phosphorylation de la tyrosine, de la fixation des domaines SH2 de la sous-unité p85 de la phosphoinositide 3-kinase (PI3K) et de Grb2 et GADS (Grb2-related adaptor downstream of Shc) $[10,11]$. Deux motifs riches en proline, PRRP et PYAP, recrutent les domaines SH3 des PTK (protéine tyrosine kinase) Tec et Itk (PRRP) et Lck et Grb2 (PYAP) ainsi que la filamine A (FLNA) [11]. L'activité PI3K régule des fonctions comme la progression dans le cycle cellulaire, l'inhibition de l'apoptose, le métabolisme cellulaire et la production de cytokines [12, 13]. Cependant, le recrutement de Grb2 et des PTK participe à la régulation des petites protéines GTPases et de réarrangements du cytosquelette [11]. La PKC $\theta$ est une molécule de signalisation «pivot » dans le mécanisme d'action de CD28, dont le rôle a été précisé chez les souris $c d 28^{-/-}$[14] (Figure 2). La PKC- $\theta$ est recrutée par $\mathrm{CD} 28$ par un mécanisme qui implique le motif PYAP au niveau de la «synapse » immune [15]. La stabilisation des ARNm codant pour les cytokines dépendrait aussi de ce motif intracytoplasmique PYAP [12].

L'ensemble des fonctions de CD28 est donc induit par une combinaison d'effets dépendants de PI3K et d'effets liés au recrutement de différentes protéine kinases/molécules adaptatrices.

\section{CTLA-4 : structure, distributions cellulaire et tissulaire}

CTLA-4 appartient comme CD28 à la superfamille des Ig [16]. Les gènes qui codent pour les deux protéines sont proches sur le chromosome 2 (q33) chez l'homme et le chromosome 1 chez la souris [16]. Comme CD28, CTLA-4 forme des homodimères et fixe les ligands CD80 et CD86 [16] par des mécanismes qui impliquent le motif MYPPPy présent dans le CDR3 (complementarity determining region 3 ) de CTLA-4 comme dans celui de CD28 [16-18]. L'affinité de l'interaction entre CTLA-4 et CD80 est cependant supérieure à celle qui existe entre CD28 et CD80 (respectivement 12 et 200 nM) [19]. Ce phénomène est lié à la capacité des homodimères de CTLA-4 de s'associer et de lier des molécules CD80 sous forme bivalente. Une forme soluble de CTLA-4 (sCTLA-4) a été décrite, dont l'expression est augmentée dans les 


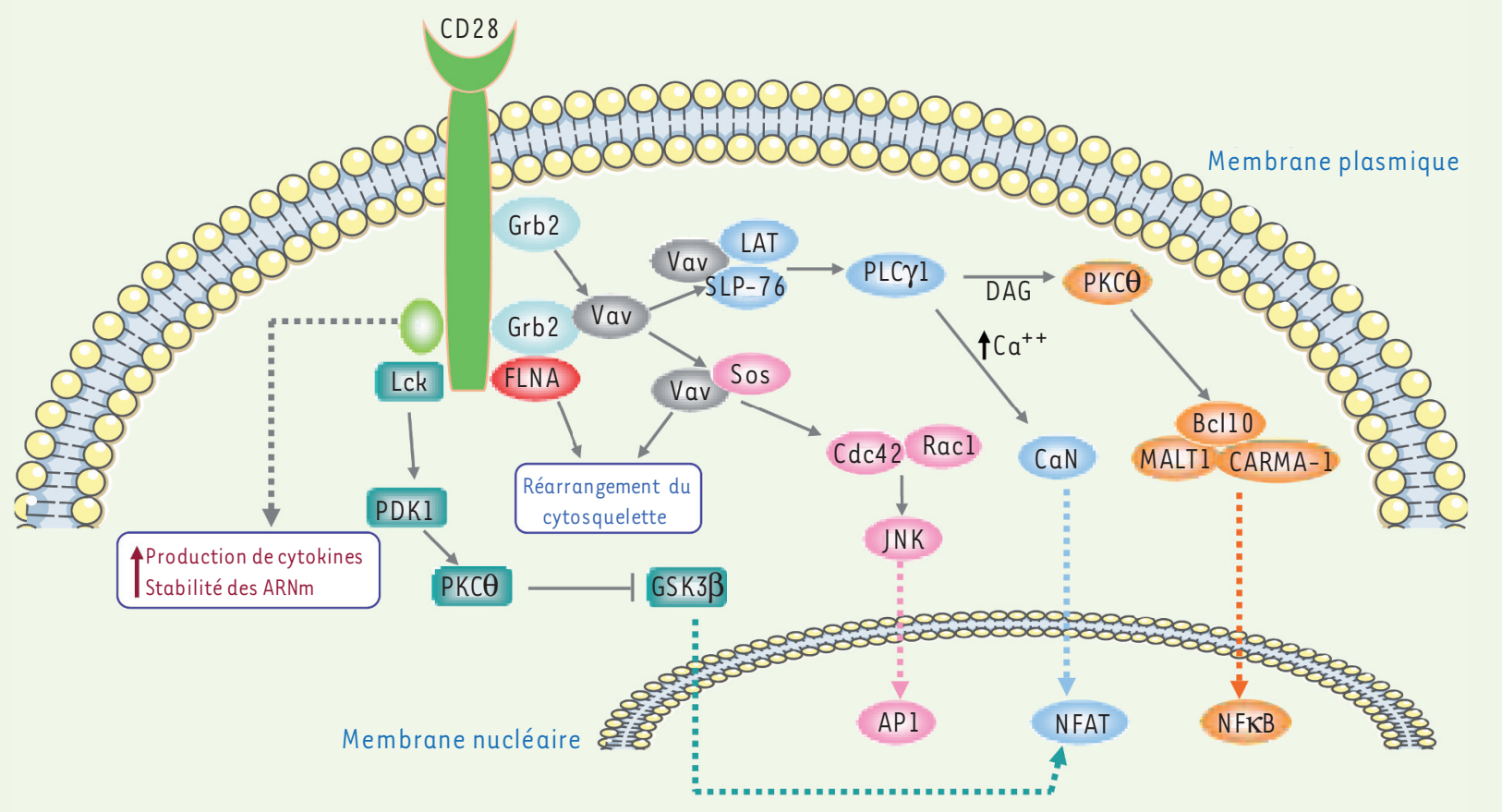

Figure 2. Pharmacologie PI3K-indépendante induite par CD28. Les différentes protéine kinase/molécules adaptatrices recrutées au niveau du domaine intracytoplasmique de CD28 sont représentées, ainsi que leurs cibles effectrices. PI3K : phosphatidylinositol 3'-kinase; Lck: lymphocyte cellular kinase ; PDK-1 : phosphoinositide-dependent protein kinase 1; PKC $\theta$ : protein kinase C $\theta$; GSK3b : glycogen synthetase kinase 3B ; Grb2 : growth factor receptor-bound protein 2 ; FLNA : filamin A; Vav : vav proto-oncogene; LAT: linker of activation in $T$ cell ; SLP-76: SH2-domain-containing leucocyte protein of 76 kDa ; PLC-gl : phospholipase c g-1; DAG : diacyl glycerol; PKC $\theta$ : protein kinase C $\theta$; CARMAl : caspase recruitment domain (CARD)-containing membrane-associated guanylate kinase (MAGUK) protein-1; Bcllo: B-cell lymphoma/leukemia 10 ; MALT1 : mucosa-associated lymphoma translocation protein 1; CaN : calcineurin; Sos : son of sevenless ; Cdc42 : cell division cycle 42 ; Racl : Ras-related C3 botulinum toxin substrate 1; JNK : jun kinase ; API : activator protein 1 ; NFAT : nuclear factor of activated T-cells; NFKB : nuclear factor kappa B.

maladies auto-immunes. CTLA-4 est localisée dans le compartiment intracellulaire dans les lymphocytes $\mathrm{T}$, associée à la protéine $T c R$ interacting protein (TRIM) dans le réseau transgolgien. Elle apparaît à la surface cellulaire des lymphocytes $T$ conventionnels après leur activation (Figure 1). Son expression à la surface cellulaire est régulée par l'activité de la phospholipase D et la GTPase ARFl. À la surface cellulaire, CTLA-4 est phosphorylée au niveau de la tyrosine du motif YVKM par les PTK. Ce motif phosphorylé serait susceptible de recruter la PI3K ou la tyrosine phosphatase SHP2. Cette phosphorylation est essentielle pour favoriser l'expression de CTLA-4 à la surface et retarder son internalisation. En effet, une déphosphorylation permet la fixation de la protéine adaptatrice AP-2 au niveau du motif GVYVKM et induit son endocytose. Son expression de surface est aussi stabilisée par son interaction avec ses ligands portés par la cellule présentatrice d'antigène.

CTLA-4 est présente de façon constitutive à la surface des lymphocytes T régulateurs (Treg), et elle a aussi été décrite à la surface d'autres cellules immunes [20] et non immunes. Dans ce cadre, sa fonction reste inconnue, mais pourrait être importante en particulier pour les effets thérapeutiques des Acm.

\section{Fonction de CTLA-4 : un frein (co-inhibiteur)}

CTLA-4 régule de multiples facettes des fonctions immunes [16]. Son rôle de régulateur négatif des fonctions immunes a été démontré par l'étude des souris ctla-4 $4^{-/-}$et I'utilisation d'Acm. Les souris ctla-4/-/- développent une activation et une prolifération massive des lymphocytes T. L'absence de CTLA-4 se traduit en particulier par une expansion des T CD4 (Figure 3 ). Les souris meurent de maladies vraisemblablement auto-immunes systémiques associées à une infiltration de lymphocytes $T$ activés de façon précoce dès la troisième semaine après leur naissance $[21,22]$. Ces effets sont dépendants de la présence de CD28 et donc vraisemblablement de la costimulation qu'induit cette molécule. Les effets des Acm anti-CTLA-4, depuis les travaux pionniers de P.S. Linsley jusqu'aux données actuelles, montrent que CTLA-4 est une molécule qui inhibe les fonctions immunes et régule l'expansion des effecteurs T CD8 ${ }^{+}$et le développement de cellules mémoires [23, 24]. 


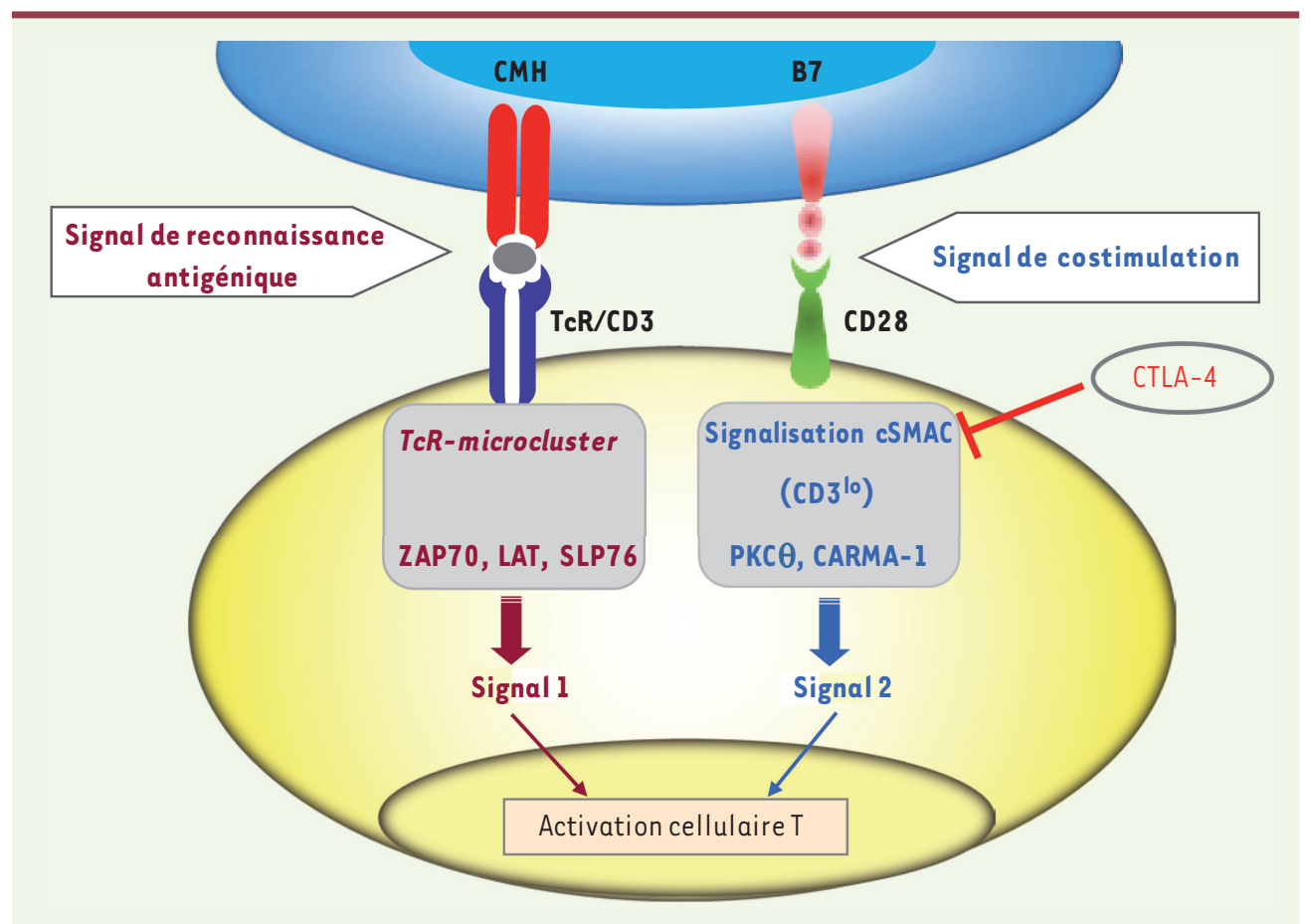

Figure 3. Fonction inhibitrice de CTLA-4. Dans un contexte de reconnaissance antigénique, les signaux de costimulation via CD28 (ici PI3K-indépendant) peuvent être inhibés par l'intervention de CTLA-4. ZAP-70 : zeta-chainassociated protein kinase of $70 \mathrm{kDa}$; LAT : linker of activation in T cell ; SLP-76 : SH2-domain-containing leukocyte protein of $76 \mathrm{kDa}$; PKC $\theta$ : protein kinase C $\theta$; CARMAl : caspase recruitment domain (CARD)-containing membrane-associated guanylate kinase (MAGUK) protein-1; CSMAC : central supramolecular activation cluster.

\section{CTLA-4 est un} compétiteur de la fonction de costimulation de CD28 Plusieurs approches démontrent cette compétition : (1) des expériences de compétition pour l'accessibilité et la fixation des ligands communs à CD28 et CTLA-4 que sont CD80 et CD86, et (2) les données qui montrent une compétition pour la localisation au niveau de la zone de contact ou « synapse immune » entre les cellules $T$ et les cellules présentatrices d'antigène. La partie centrale de la synapse (ou cSMAC, central supramolecular activation cluster) contient le TcR du côté T et les molécules du complexe majeur d'histocompatibilité (CMH) côté cellules présentatrices d'antigène ; les molécules d'adhé-

Dans certains modèles murins, les fonctions de CTLA-4 sur les Treg (expression constitutive de CTLA-4) et les T conventionnels (seuls les T activés expriment (TLA-4) ont pu être dissociées grâce à l'utilisation de souris qui expriment le transgène ctla-4 sous le contrôle du promoteur du gène $\| l-2$ chez des souris ctla- $4^{-/-}$. Ce promoteur ne s'exprimant pas dans les Treg, CTLA-4 est donc exclusivement exprimé dans les lymphocytes T effecteurs et pas dans les Treg [25]. Ce modèle indique que CTLA-4 porté par les Treg est importante pour réguler les fonctions immunosuppressives des Treg en association au TGF $\beta$ (transforming growth factor) et à I'IL-10. Par ce biais, les Treg vont réguler l'activation des $T$ conventionnels aux antigènes du soi et de l'environnement. Dans d'autres modèles de tolérance aux antigènes du soi d'origine pancréatique, les molécules CTLA-4 exprimées sur les deux types de lymphocytes, Treg et T conventionnels, collaborent pour préserver la tolérance au soi [26].

\section{Mécanismes impliqués dans les fonctions de CTLA-4}

Les données in vitro et in vivo suggèrent que les mécanismes d'immunosuppression interviennent à différents niveaux : (1) compétition pour la costimulation par CD28, (2) régulation de la signalisation cellulaire par l'intermédiaire de phosphatases, (3) signaux activateurs propres apportés par CTLA-4. rence comme LFA-1 (leukocyte function-associated, de la famille des intégrines $\beta 2$ ) et ICAM (intercellular adhesion molecule) sont situées en périphérie (pSMAC) [27]. Les molécules de costimulation et de cosignalisation négative se localisent également au niveau de la synapse immune. L'activation $T$ et la costimulation se traduisent par la formation de microclusters correspondant au TcR associé à des molécules de signalisation (ZAP-70, SLP-76). La costimulation par CD28 recrute la PKC- $\theta$ [28]. Au sein du CSMAC, deux sous-régions seraient identifiées qui se distinguent par la densité en TcR-CD3 : les régions de haute et basse densité en TcR-CD3 (CD3hi et CD3lo). Cette dernière région recrute $\operatorname{CD} 28$ et $\mathrm{PKC}-\theta$ [28]. Pendant l'activation, CTLA-4 se trouve localisée au niveau de la région CD3lo de la synapse immune, où il entre en compétition avec le recrutement de $C D 28$, empêchant ainsi le recrutement de la PKC- $\theta$ au niveau de la synapse immune [29] (Figure 3). Ces mécanismes sont à rapprocher de ceux qu'utilise CTLA-4 dans la régulation de la formation de radeaux (rafts) et le recrutement de ZAP-70. De même, l'inhibition des signaux, dits stop, induits par le TcR et décrits par le groupe de C. Rudd pourraient dépendre de mécanismes semblables d'inhibition d'une signalisation cellulaire efficace via la prévention de la costimulation CD28 [11]. 


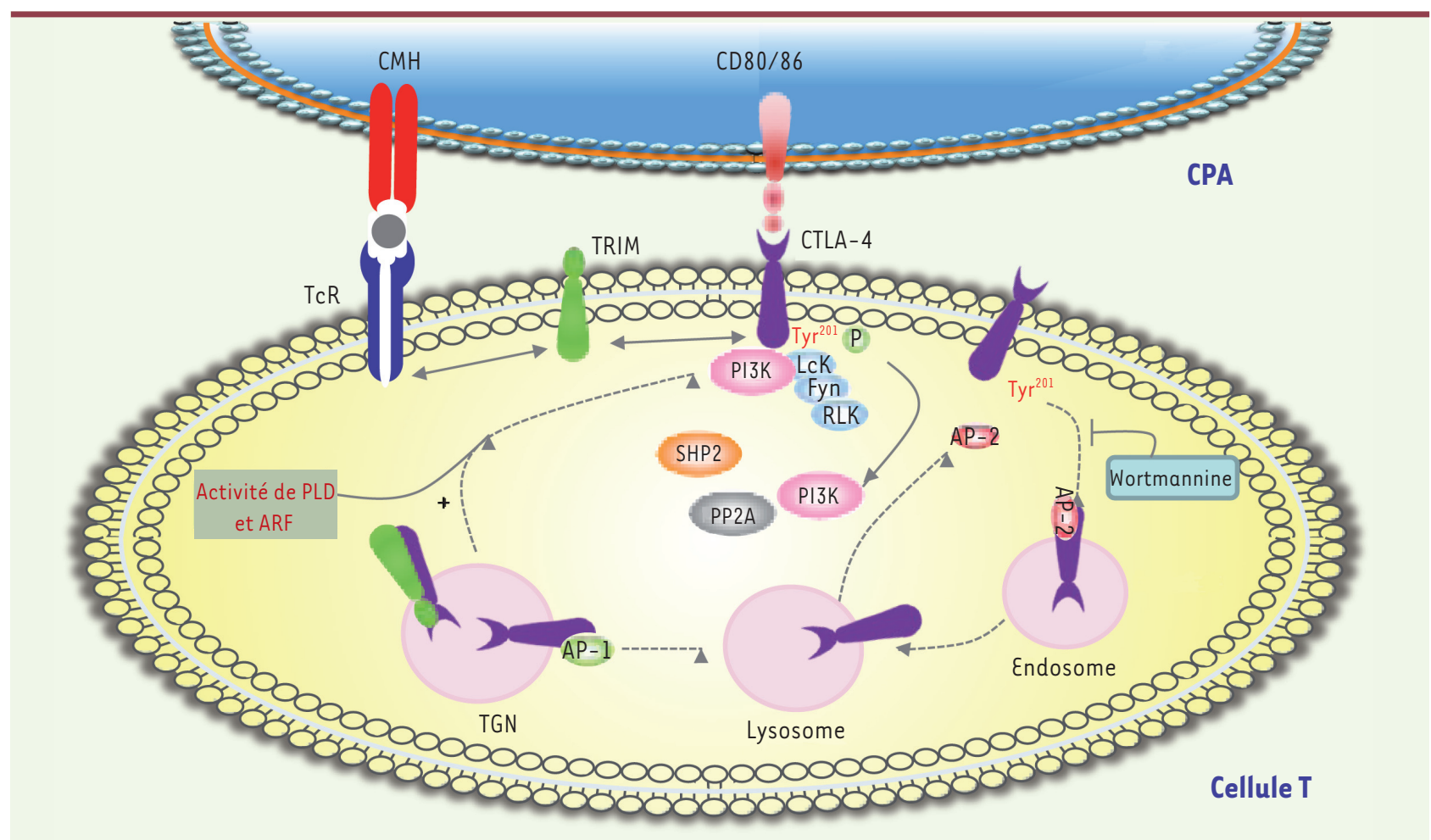

Figure 4. Pharmacologie de l'action induite par le récepteur CTLA-4. CPA : cellule présentatrice d'antigène ; TRIM : T-cell receptor-interacting molecule ; TGN : trans-Golgi network ; PLD : phospholipase D ; ARF : adenosine diphosphate (ADP)-ribosylation factor; AP : adapter protein ; PI3K : phosphatidylinositol 3'-kinase; SHP2 : SH2-domain-containing protein tyrosine phosphatase 1; PP2A : protein phosphatase 2A; Lck : lymphocyte cellular kinase; Fyn: Fgr/yes-related novel gene; RLK : receptor-like kinases (d'après [11]).

Le rôle du domaine intracytoplasmique (IC)

de CTLA-4 dans ses fonctions immunosuppressives

\section{a été proposé}

Les arguments princeps sont basés sur la démonstration qu'un transgène correspondant au gène ctla-4 dépourvu de son domaine IC ne corrige pas le phénotype des souris $c t l a-4^{-/-}$. Ces données indiquent que le domaine IC de CTLA-4 et donc les seconds messagers que ce domaine contrôle, sont importants pour sa fonction. Les motifs intracytoplasmiques YVKM et riches en lysines du domaine IC de CTLA-4 sont impliqués dans l'interaction avec les phosphatases Src homology 2 (SH2)-domain containing tyrosine phosphatase 1 et 2 (SHP1 et SHP2) et PP2A respectivement [11]. La visualisation directe de l'interaction entre ces phosphatases et CTLA-4, et leurs fonctions, restent à préciser.

\section{CTLA-4 induit des signaux intracellulaires}

Ces signaux intracellulaires régulent des activités kinase comme la PI3K et JNK sans que les bases structurales n'en soient clairement apportées (Figure 4) [11].

Pour conclure, l'engagement de CTLA-4 se traduit par une inhibition de l'activation des lymphocytes $\mathrm{T} \mathrm{CD4}^{+}$et de leur progression dans le cycle cellulaire. CTLA-4 pourrait augmenter le seuil d'activation des lymphocytes T et donc les mécanismes d'auto-immunité liés aux antigènes du soi de faible affinité. En dehors de celles qui s'exercent dans les lymphocytes $T$, d'autres fonctions de CTLA-4 restent à découvrir. Citons par exemple son rôle dans les cellules dendritiques matures, puisque des Acm anti-CTLA- 4 induisent la production d'IL-10 et inhibent la production d'IL-8 par ces cellules [20].

\section{CTLA- 4 et modèles précliniques}

Dès 1996, le laboratoire de J. Allison démontrait que les Acm anti-CTLA-4 utilisés en monothérapie induisaient l'élimination de tumeurs [27]. Dans certains modèles, le rejet des tumeurs transplantées permettait d'induire une protection contre une autre greffe tumorale, indiquant la génération d'une immunité antitumorale dotée d'une mémoire. Les données précliniques montrent que l'antiCTLA-4 utilisé seul est capable d'induire l'élimination de tumeurs immunogéniques (suscitant une réaction immune). Cependant, les tumeurs peu ou non immunogéniques nécessitent la mise en place des thérapies combinées avec du GM-CSF (granulocyte-macrophage colony-stimulating factor) ou avec des vaccins thérapeutiques, la déplétion des 


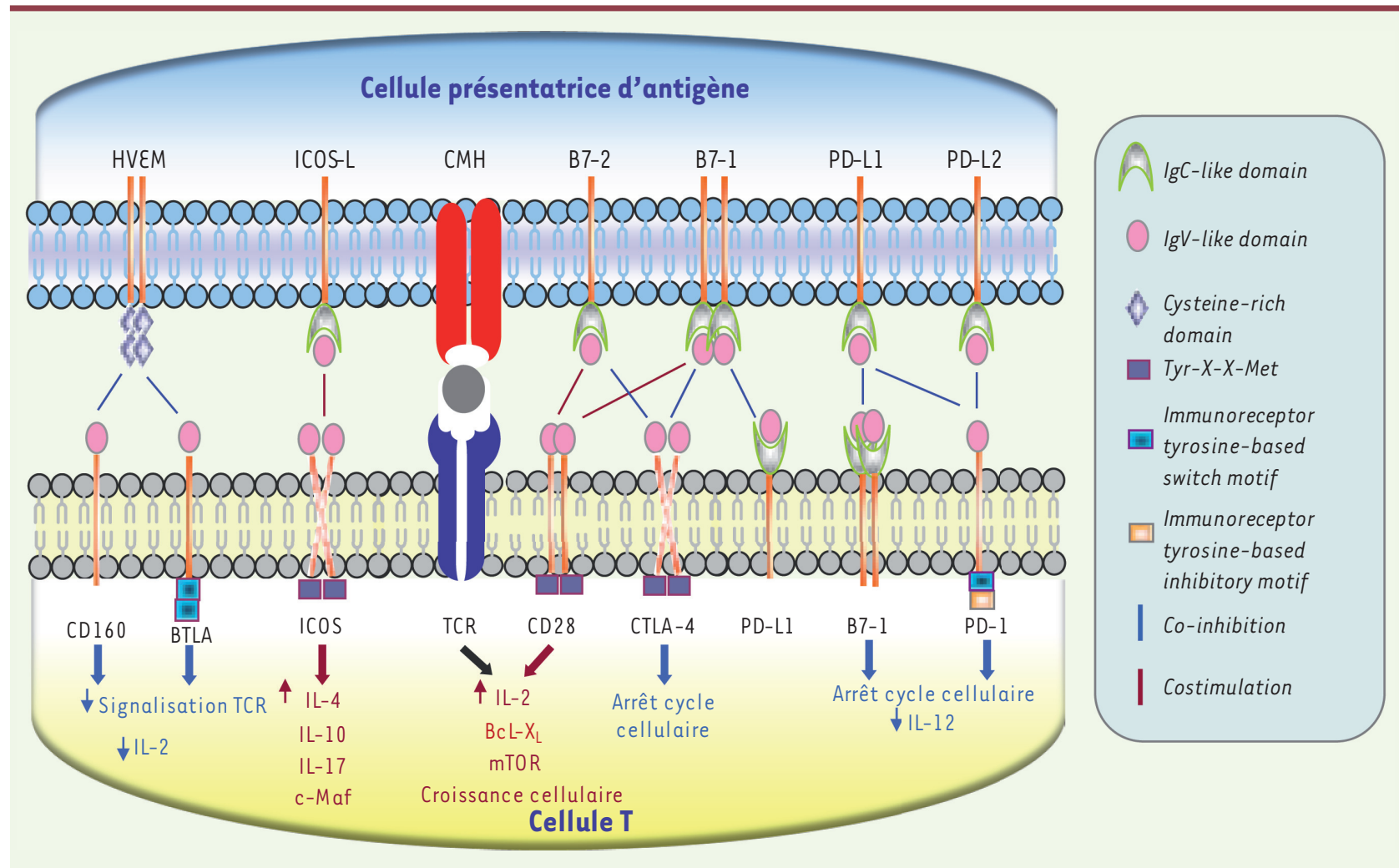

Figure 5. Les mécanismes de cosignalisation des lymphocytes T. HVEM : herpes virus entry mediator; ICOS : inducible costimulator; BTLA : B and T lymphocyte attenuator; PD- 1 : programmed death-1 ; mTOR : mammalian target of rapamycin; BcL-X : longer alternatively spliced form of BcL-x. B7-1 représente CD80, et B7-2 CD86.

Treg, des chimiothérapies ou l'association à d'autres immunothérapies (anti-PD-1 [programmed death-1]) [30]. La régression tumorale est bien induite par le système immunitaire puisque les effets de l'anti-CTLA-4 sont perdus chez les souris dépourvues de lymphocytes T. Comme CTLA-4 est exprimée constitutivement sur les cellules Treg, il a été proposé que l'élimination de la suppression induite par les Treg expliquait l'activité thérapeutique des anti-CTLA-4. Les données actuelles ne sont pas en faveur de cette hypothèse, mais plutôt en faveur de modifications du rapport cellules effectrices sur cellules Treg, en particulier dans la tumeur.

Les modèles murins privilégient le rôle combiné des $T \operatorname{CD} 8^{+}$et des $T$ $\mathrm{CD}_{4}{ }^{+}$dans l'effet thérapeutique des anti-CTLA-4. Les modèles animaux ont montré que peu de pathologies auto-immunes étaient associées au traitement anti-CTLA-4 hormis un vitiligo auto-immun dans le modèle non immunogénique B16 [31]. Ces données ne préfiguraient que partiellement les données cliniques observées chez les patients traités par antiCTLA-4, qui sont décrites ci-dessous, ainsi que par C. Mateus, page 850 C. Robert et C. Matteus [37] $(\rightarrow)$.

$\rightarrow$ Voir l'article de C. Robert et de ce numéro

Dans le cadre des modèles animaux de réponses antitumorales, les $T$ conventionnels, et non les Treg, sont les acteurs préférentiels de l'action des anti-CTLA-4.

\section{CTLA-4 et les autres mécanismes de cosignalisation}

La découverte de nombreuses molécules de cosignalisation après CTLA-4 peut sembler surprenante. Mais les données d'invalidation génique chez la souris indiquent que ces molécules (CTLA-4, PD-1, BTLA [ $B$ and T lymphocyte attenuator], HVEM [herpes virus entry mediator]) régulent les mécanismes de tolérance du soi et d'auto-immunité avec des cinétiques et des intensités différentes [32, 33] (Figure 5). Les données phénotypiques obtenues in vitro ou in vivo chez des patients atteints d'infections virales chroniques ou de cancers indiquent que les cellules immunes activées peuvent coexprimer différentes molécules de cosignalisation négatives comme CTLA- 4 mais aussi PD-1, BTLA, LAG3, CD160 et Tim-3. Ces molécules sont activées par des ligands différents mais qui peuvent être coexprimés par les mêmes cellules, cellules présentatrices d'antigène ou cellules tumorales. Les données fonctionnelles démontrent une coopération des molécules correspondantes. 
Cette régulation croisée a, dans certains cas, des bases biochimiques. Ainsi, si CD80 est le ligand de CD28 et CTLA-4, il est aussi celui de $P D-1$. Ces données suggèrent donc une possible régulation croisée par ces molécules de cosignalisation. Ces paramètres montrent, s'il en était besoin, la subtilité des régulations immunes et la difficulté de prédire dans un mode binaire leurs conséquences dans des modèles physiologiques.

BTLA diffère des molécules de cosignalisation « classiques », c'està-dire régulées positivement par l'activation et la différenciation cellulaires, par son expression préférentielle sur les cellules T naïves $[34,35]$.

Les signaux intracellulaires impliqués sont distincts de ceux impliquant CTLA-4. Par exemple, PD-1 et BTLA induisent leurs fonctions via des motifs de type ITIM (immunoreceptor tyrosine-based inhibitory motif) et ITSM (immunoreceptor based switch motif) retrouvés dans leur domaine intracytoplasmique.

Les conséquences fonctionnelles des blocages combinés ont été évaluées dans des modèles précliniques, en particulier dans le cas de PD-1 et CTLA-4. Une augmentation de l'infiltration des cellules T effectrices et de leurs capacités fonctionnelles a été observée dans un de ces modèles [30].

\section{Mécanismes d'action in vivo et prédictifs de réponses cliniques}

Les mécanismes d'action des Acm anti-CTLA-4 in vivo reposent sur le fait que ces Acm, qui inhibent l'interaction entre CTLA-4 et ses ligands, devraient prévenir les signaux induits par CTLA-4 quels qu'ils soient. Les observations chez les patients traités par les Acm thérapeutiques indiquent que le ciblage de CTLA-4 se traduit par des manifestations inflammatoires qui indiquent une activation immune $[36,37]$. Les effets secondaires correspondent à des atteintes de type colite, prurit, hypothyroïdie, nécrose hypophysaire $\rightarrow$ [37]).

$\rightarrow$ Voir l'article de C. Robert et C. Mateus, page 850 de ce numéro Les données cliniques indiquent que les réponses cliniques sont associées aux manifestations immunes pouvant conduire à l'autoimmunité. Ces données confirment que les réponses immunes et/ou inflammatoires, sans que le rôle des lymphocytes T puisse être spécifiquement montré, peuvent contrôler un cancer avancé jusqu'à induire une réponse clinique complète. Les paramètres biologiques de réponse aux Acm de type anti-CTLA-4 sont un enjeu important. Ils doivent pouvoir sélectionner les patients afin: (1) d'éviter des effets secondaires parfois délétères, (2) de permettre de proposer des combinaisons thérapeutiques chez les patients résistants, en particulier par d'autres « inhibiteurs d'inhibiteurs » comme des Acm anti-PD-1 ou des thérapies complémentaires.

Les paramètres associés à la réponse restent peu discriminants. Ils correspondent à l'augmentation des lymphocytes, en particulier de l'infiltrat lymphocytaire intratumoral et de marqueurs d'activation comme ICOS (inducible costimulator) ainsi qu'à la diminution d'autres facteurs immunosuppresseurs tels que l'expression de FoxP3 ou d'indoleamine 2,3-dioxygénase (IDO).

\section{Les frontières}

Les questions sur les fonctions de CTLA-4 et sur son utilisation thérapeutique restent nombreuses. Parmi celles-ci, on peut indiquer les fonctions de CTLA-4 : (1) sur des cellules différenciées au-delà des $T$ activés et des Treg, en particulier les Th17, $\mathrm{T}_{\mathrm{FH}}{ }^{2}$ et les autres sous-populations effectrices en cours de démembrement; (2) sur les autres cellules immunes « non $T$ » et non immunes et leur rôle dans l'action des Acm thérapeutiques.

L'analyse détaillée des coopérations des voies de cosignalisation est la suite logique de la démonstration de leur coopération dans des modèles in vitro et précliniques [30]. On commencera très bientôt à tirer parti de ces connaissances, en particulier dans les formes tumorales résistantes aux anti-CTLA-4. Du point de vue pharmacologique, la dissection des voies métaboliques et événements cellulaires régulés par CTLA-4 reste un objectif important pour enrichir nos stratégies thérapeutiques. $\diamond$

\section{SUMMARY}

The role of co-inhibitory signals driven by CTLA-4 in immune system

Key players on the immunosurveillance program, T cells are regulated by their surface receptors such as $T$-cell receptor (TCR), and costimulatory molecules, optimizing T-cell activation. Some of these costimulatory molecules, such as the cytotoxic T-lymphocyte antigen 4 (CTLA4), induce inhibitory effects on T cells. By "inhibiting the inhibitor" CTLA-4-blocking monoclonal antibodies represent a novel class of weapons against cancers. To better understand the promising future and the limits of this immunotherapy, this review dissects the molecular inhibitory mechanisms induced by CTLA-4 in T cells. $\diamond$

\section{CONFLIT D'INTÉRÊTS}

Les auteurs déclarent n'avoir aucun conflit d'intérêts concernant les données publiées dans cet article.

\section{RÉFÉRENCES}

1. Schwartz RH. Costimulation of T lymphocytes: the role of CD28, CTLA-4, and $\mathrm{B} 7 / \mathrm{BB} 1$ in interleukin-2 production and immunotherapy. Cell $1992 ; 71$ : 1065-8.

2. Linsley PS, Ledbetter JA. The role of the CD28 receptor during T cell responses to antigen. Annu Rev Immunol 1993; 11 : 191-212.

3. Thompson CB, Lindsten T, Ledbetter JA, et al. CD28 activation pathway regulates the production of multiple T-cell-derived lymphokines/cytokines. Proc Natl Acad Sci USA $1989 ; 86: 1333-7$.

${ }^{2}$ Les $T_{F H}$ sont des cellules T CD 4 effectrices appelées lymphocytes T CD4 folliculaires (TFH) qui contrôlent le développement et la maturation des cellules $B$ spécifiques de l'antigène dans les centres germinatifs. 
4. Linsley PS, Brady W, Grosmaire L, et al. Binding of the B cell activation antigen B7 to CD28 costimulates T cell proliferation and interleukin 2 mRNA accumulation. J Exp Med 1991 ; $173: 721-30$.

5. Cerdan C, Martin Y, Courcoul M, et al. CD28 costimulation up-regulates long-term IL-2R beta expression in human $\mathrm{T}$ cells through combined transcriptional and post-transcriptional regulation. J Immunol 1995 ; 154 : 1007-13.

6. Boise LH, Minn AJ, Noel PJ, et al. CD28 costimulation can promote T cell survival by enhancing the expression of Bcl-XL. Immunity $1995 ; 3: 87-98$.

7. Tacke M, Hanke G, Hanke T, Hunig T. CD28-mediated induction of proliferation in resting T cells in vitro and in vivo without engagement of the T cell receptor: evidence for functionally distinct forms of CD28. Eur J Immunol 1997 ; 27 : 239-47.

8. Fraser JD, Irving BA, Crabtree GR, Weiss A. Regulation of interleukin-2 gene enhancer activity by the T cell accessory molecule CD28. Science $1991 ; 251$ : 313-6.

9. Granelli-Piperno A, Nolan P. Nuclear transcription factors that bind to elements of the IL-2 promoter. Induction requirements in primary human T cells. J Immunol 1991 ; 147 : 2734-9.

10. Pages F, Ragueneau M, Rottapel R, et al. Binding of phosphatidylinositol-3-OH kinase to CD28 is required for T-cell signalling. Nature $1994 ; 369: 327-9$.

11. Rudd CE, Taylor A, Schneider H. CD28 and CTLA-4 coreceptor expression and signal transduction. Immunol Rev $2009 ; 229$ : 12-26.

12. Sanchez-Lockhart M, Marin $\varepsilon$, Graf B, et al. Cutting edge: CD28-mediated transcriptional and posttranscriptional regulation of IL-2 expression are controlled through different signaling pathways. J Immunol 2004 ; $173: 7120-4$.

13. Miller J, Baker C, Cook K, et al. Two pathways of costimulation through CD28. Immunol Res 2009; 13 février (online).

14. Huang J, Lo PF, Zal T, et al. CD28 plays a critical role in the segregation of PKC theta within the immunologic synapse. Proc Natl Acad Sci USA 2002 ; 99 : 9369-73.

15. Saito T, Yokosuka T, Hashimoto-Tane A. Dynamic regulation of T cell activation and co-stimulation through TCR-microclusters. FEBS Lett $2010 ; 584: 4865-71$.

16. Linsley PS, Golstein P. Lymphocyte activation: T-cell regulation by CTLA-4. Curr Biol $1996 ; 6$ : 398 400.

17. Peach RJ, Bajorath J, Brady W, et al. Complementarity determining region I (CDRI)- and CDR3analogous regions in CTLA-4 and CD28 determine the binding to B7-1.J Exp Med $1994 ; 180$ : 2049-58.

18. Truneh A, Reddy M, Ryan $P$, et al. Differential recognition by CD28 of its cognate counter receptors CD80 (B7.1) and B70 (B7.2): analysis by site directed mutagenesis. Mol Immunol 1996 ; $33: 321-34$

19. Van der Merwe PA, Bodian DL, Daenke S, et al. CD80 (B7-1) binds both CD28 and CTLA-4 with a low affinity and very fast kinetics. J Exp Med 1997 ; 185 : 393-403.

20. Laurent S, Carrega P, Saverino D, et al. CTLA-4 is expressed by human monocyte-derived dendritic cells and regulates their functions. Hum Immunol $2010 ; 71: 934-41$

21. Tivol EA, Borriello F, Schweitzer AN, et al. Loss of CTLA-4 leads to massive lymphoproliferation and fatal multiorgan tissue destruction, revealing a critical negative regulatory role of CTLA-4. Immunity $1995 ; 3: 541-7$

22. Waterhouse $P$, Penninger JM, Timms $\varepsilon$, et al. Lymphoproliferative disorders with early lethality in mice deficient in Ctla-4. Science $1995 ; 270: 985-8$

23. Walunas TL, Lenschow DJ, Bakker CY, et al. CTLA-4 can function as a negative regulator of T cell activation. Immunity $1994 ; 1: 405-13$.
24. Pedicord VA, Montalvo W, Leiner IM, Allison JP. Single dose of anti-CTLA-4 enhances CD8 ${ }^{+} \mathrm{T}$-cell memory formation, function, and maintenance. Proc Natl Acad Sci USA $2010 ; 108: 266-71$.

25. Jain N, Nguyen H, Chambers C, Kang J. Dual function of CTLA-4 in regulatory $T$ cells and conventional T cells to prevent multiorgan autoimmunity. Proc Natl Acad Sci USA 2010 ; 107 : 1524-8.

26. Ise W, Kohyama M, Nutsch KM, et al. CTLA-4 suppresses the pathogenicity of self antigen-specific $T$ cells by cell-intrinsic and cell-extrinsic mechanisms. Nat Immunol 2010; 11: 129-35

27. Dustin ML. Insights into function of the immunological synapse from studies with supported planar bilayers. Curr Top Microbiol Immunol $2010 ; 340$ : 1-24.

28. Yokosuka T, Kobayashi W, Sakata-Sogawa K, et al. Spatiotemporal regulation of T cell costimulation by TCR-CD28 microclusters and protein kinase $\mathrm{C}$ theta translocation. Immunity 2008 ; 29 : 589-601.

29. Yokosuka T, Kobayashi W, Takamatsu M, et al. Spatiotemporal basis of CTLA4 costimulatory molecule-mediated negative regulation of $\mathrm{T}$ cell activation. Immunity $2010 ; 33: 326-39$.

30. Curran MA, Montalvo W, Yagita H, Allison JP. PD- 1 and CTLA-4 combination blockade expands infiltrating $T$ cells and reduces regulatory $T$ and myeloid cells within B16 melanoma tumors. Proc Natl Acad Sci USA 2010; 107 : 4275-80.

31. Van Elsas A, Hurwitz AA, Allison JP. Combination immunotherapy of B16 melanoma using anti-cytotoxic T lymphocyte-associated antigen 4 (CTLA4) and granulocyte/macrophage colony-stimulating factor (GM-CSF)producing vaccines induces rejection of subcutaneous and metastatic tumors accompanied by autoimmune depigmentation. J Exp Med $1999 ; 190$ : 355-66.

32. Sharpe AH. Mechanisms of costimulation. Immunol Rev $2009 ; 229: 5-11$.

33. Olive D. Corécepteurs lymphocytaires. Med Sci (Paris) 2006 ; 22 : 1069-74.

34. Watanabe N, Gavrieli M, Sedy JR, et al. BTLA is a lymphocyte inhibitory receptor with similarities to CTLA-4 and PD-1. Nat Immunol $2003 ; 4: 670-9$.

35. Serriari NE, Gondois-Rey F, Guillaume $Y$, et al. B and T lymphocyte attenuator is highly expressed on CMV-specific T cells during infection and regulates their function. J Immunol $2010 ; 185: 3140-8$.

36. Weber $J$. Review: anti-CTLA-4 antibody ipilimumab: case studies of clinica response and immune-related adverse events. Oncologist $2007 ; 12: 864-72$.

37. Robert C, Matteus C. Anticorps anti-CTLA-4: une avancée thérapeutique majeure dans le traitement du mélanome métastatique. Med Sci (Paris) $2011 ; 27: 850-8$.

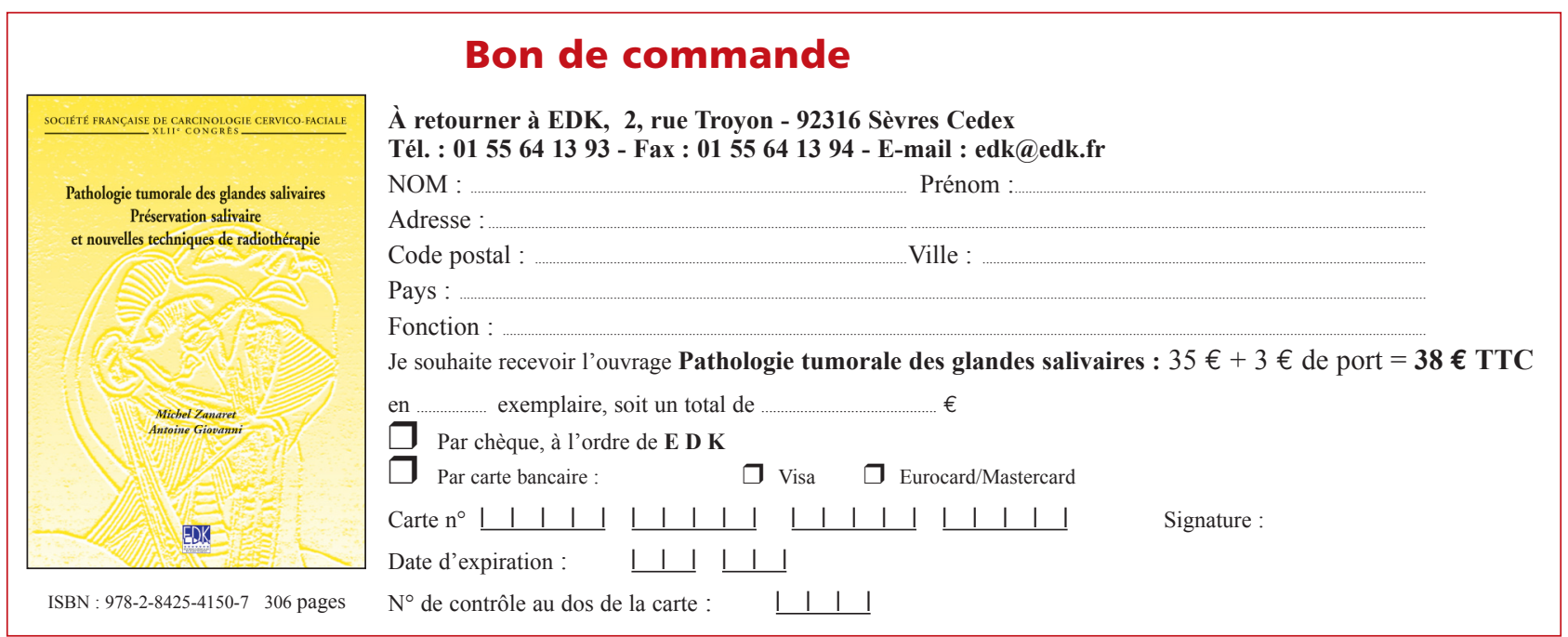

